\title{
ON THE OSCILLATION OF SOLUTIONS OF CERTAIN LINEAR DIFFERENTIAL EQUATIONS IN THE COMPLEX DOMAIN
}

\author{
by STEVEN B. BANK and J. K. LANGLEY
}

(Received 24th March 1986)

\section{Introduction}

Our starting point is the differential equation

$$
y^{\prime \prime}+A(z) y=0
$$

where $A(z)$ is a transcendental entire function of finite order, and we are concerned specifically with the frequency of zeros of a non-trivial solution $f(z)$ of $(1.1)$. Of course it is well known that such a solution $f(z)$ is an entire function of infinite order, and using standard notation from [7],

$$
N\left(r, \frac{1}{f-b}\right) \sim T(r, f)
$$

for all $b \in C \backslash\{0\}$, at least outside a set of $r$ of finite measure. The same conclusions hold if $y^{\prime \prime}$ is replaced by a higher derivative in (1.1). Denoting by $\sigma(g)$ the order of an entire function $g$, and by $\lambda(g)$ the exponent of convergence of its zeros we have the following, proved in $[1,3]$ :

Theorem A. Let $A(z)$ be a transcendental entire function, and let $f_{1}, f_{2}$ be linearly independent solutions of (1.1).

(a) Suppose that $\sigma(A)$ is finite but is not a positive integer. Then $\max \left\{\lambda\left(f_{1}\right), \lambda\left(f_{2}\right)\right\}$ is not less than $\sigma(A)$, and is infinite if $\sigma(A)<\frac{1}{2}$.

(b) Suppose that $\lambda(A)<\sigma(A)<\infty$.

Then for any $k \geqq 2$ and any non-trivial solution $f(z)$ of

$$
y^{(k)}+A(z) y=0
$$

we have $\lambda(f) \geqq \sigma(A)$.

We remark that it is conjectured that under the hypotheses of Theorem $\mathbf{A}$, part (a), we always have

$$
\max \left\{\lambda\left(f_{1}\right), \lambda\left(f_{2}\right)\right\}=\infty
$$


The following result was proved in [4], and gives conditions under which this stronger conclusion holds:

Theorem B. Let $A(z)$ be a transcendental entire function of finite order $\rho$ with the following property: there exists a set $H \subseteq \mathbb{B}$ of measure zero such that for each real $\theta$ not in $\mathrm{H}$ either

$$
\begin{gathered}
r^{-N}\left|A\left(r e^{i \theta}\right)\right| \rightarrow \infty \text { as } r \rightarrow+\infty \text { for each } N>0, \text { or } \\
\int_{0}^{\infty} r\left|A\left(r e^{i \theta}\right)\right| d r<+\infty \text {, or }
\end{gathered}
$$

(iii) there exist positive real numbers $K$ and $b$, and a non-negative real number $n$ (all possibly depending on $\theta$ ), such that $(n+2)<2 \rho$ and

$$
\left|A\left(r e^{i \theta}\right)\right| \leqq K r^{n} \quad \text { for all } r \geqq b .
$$

Then if $f_{1}$ and $f_{2}$ are two linearly independent solutions of

$$
y^{\prime \prime}+A(z) y=0
$$

we have

$$
\max \left\{\lambda\left(f_{1}\right), \lambda\left(f_{2}\right)\right\}=\infty
$$

This result is sharp in that (see [4]) there exist pairs of polynomials $P(z), Q(z)$, whose degrees $d_{P}, d_{Q}$ respectively satisfy $d_{Q}+2=2 d_{P}$, such that the equation

$$
y^{\prime \prime}+\left(e^{P}+Q\right) y=0
$$

has two linearly independent non-vanishing solutions. We mention two corollaries of Theorem B.

Corollary A. Suppose that $A(z)$ is an entire function of finite order with zero as a Borel exceptional value. Then given any two linearly independent solutions $f_{1}, f_{2}$ of

$$
y^{\prime \prime}+A(z) y=0
$$

we have

$$
\max \left\{\lambda\left(f_{1}\right), \lambda\left(f_{2}\right)\right\}=\infty
$$

Corollary B. Suppose that $P(z)$ is a non-constant, even polynomial with real coefficients and with positive leading coefficient. Then all non-trivial solutions $f$ of

$$
y^{\prime \prime}+e^{P} y=0
$$


satisfy

$$
\lambda(f)=\infty
$$

Corollary B is obtained in [4] by coupling Theorem B with the Sturm theory for oscillation of real solutions of linear differential equations on the real line. The following problem is posed in [4], with reference to Corollary $B$ : if $P(z)$ is any non-constant polynomial, must every non-trivial solution $f(z)$ of

$$
y^{\prime \prime}+e^{P} y=0
$$

satisfy $\lambda(f)=\infty$ ? In the present paper we settle this problem and rather more, and our methods extend to higher order equations and have a bearing on Corollary A. We shall prove:

Theorem. Suppose that $k \geqq 2$ and that $A(z)=\Pi(z) e^{P(z)} \not \equiv 0$ where the entire function $\Pi(z)$ and the polynomial $P(z)=a_{n} z^{n}+\cdots+a_{0}$ satisfy:

(i) $\sigma(\Pi)<n$;

(ii) there exists $\theta_{0} \in \mathbb{R}$ with $\delta\left(P, \theta_{0}\right)=\operatorname{Re}\left(a_{n} e^{i n \theta_{0}}\right)=0$ and a positive $\varepsilon$ such that $\Pi(z)$ has only finitely many zeros in

$$
\left|\arg z-\theta_{0}\right|<\varepsilon
$$

Then if $n \geqq 2$ and $Q$ is a polynomial whose degree $d_{Q}$ satisfies

$$
d_{Q}+k<k n
$$

all non-trivial solutions $f$ of

$$
y^{(k)}+(A(z)+Q(z)) y=0
$$

satisfy $\lambda(f)=\infty$. The same conclusion holds if $n=1$ and $Q$ is identically zero.

We remark that the theorem is sharp at least in the case $k=2$ in view of the examples mentioned after Theorem B. We do not know if condition (ii) is sharp; its presence serves to facilitate certain asymptotic representations for the solutions of (1.2). However we do have the following corollary to our theorem:

Corollary. If $A(z)$ is a transcendental entire function of finite order having finitely many zeros, all non-trivial solutions of

$$
y^{(k)}+A(z) y=0
$$


satisfy

$$
\lambda(y)=\infty, \text { for any } k \geqq 2 .
$$

The authors would like to acknowledge valuable conversations with Robert Kaufman.

\section{Preliminaries}

We need the following definition.

Definition. An $R$-set is a countable union of discs whose radii have finite sum:

We remark that the union of two $R$-sets is an $R$-set and that (see Hayman [6], also [4]) the set of $\theta$ for which the ray $r e^{i \theta}$ meets infinitely many discs of a given $R$-set has measure zero.

Also, for a polynomial

$$
P(z)=(\alpha+i \beta) z^{n}+\cdots+a_{0}
$$

with $\alpha, \beta$ real, we define, for each real $\theta$,

$$
\delta(P, \theta)=\alpha \cos n \theta-\beta \sin n \theta
$$

and denote the degree of $P$ by $d_{P}$.

\section{Lemmas needed for the theorem}

Lemma 1. Assume the hypotheses of the theorem. Then there is a constant $c>0$ such that the asymptotic relation

$$
\left|\frac{A^{\prime}\left(r e^{i \theta}\right)}{A\left(r e^{i \theta}\right)}\right| \sim c r^{n-1} \quad \text { as } r \rightarrow \infty
$$

holds uniformly for all real $\theta$ satisfying $\left|\theta-\theta_{0}\right| \leqq 2 \varepsilon / 3$. In addition, for any real $\theta$ satisfying $0<\left|\theta-\theta_{0}\right| \leqq \varepsilon / 2$ the following are true:

(a) if $\delta(P, \theta)>0$, the function $\log \left|A\left(r e^{i \theta}\right)\right|$ is increasing on an interval $[\alpha(\theta),+\infty)$ and we have

$$
\left|A\left(r e^{i \theta}\right)\right| \geqq \exp \left(\frac{1}{2} \delta(P, \theta) r^{n}\right)
$$

there;

(b) if $\delta(P, \theta)<0$, the function $\log \left|A\left(r e^{i \theta}\right)\right|$ is decreasing on an interval $[\alpha(\theta),+\infty)$ and on that interval

$$
\left|A\left(r e^{i \theta}\right)\right| \leqq \exp \left(\frac{1}{2} \delta(P, \theta) r^{n}\right)
$$


Proof. We may write

$$
A(z)=\Pi_{1}(z) \exp \left(a_{n} z^{n}\right)
$$

where $\Pi_{1}(z)$ is an entire function of order less than $n$, and setting $\rho=\left(\sigma\left(\Pi_{1}\right)+n\right) / 2$ standard estimates yield the inequality

$$
|\log | \Pi_{1}(\zeta)|| \leqq R^{\rho}
$$

for all $\zeta$ on $|\zeta|=R$ and for all $R$ outside a set of finite linear measure $L$. Now, if $z=r e^{i \theta}$, with $\left|\theta-\theta_{0}\right| \leqq 2 \varepsilon / 3$ and $r$ sufficiently large, it follows from hypothesis (ii) of the theorem that all the zeros of $\Pi_{1}$ are at a distance at least $\delta|z|$ from $z$ for some fixed $\delta>0$. For such a point $z$ with $r>1$, since (3.4) must hold on some circle $|\zeta|=R$, with $R$ belonging to $[(L+2) r,(2 L+3) r]$, a routine application of the differentiated form of the PoissonJensen formula $[7$, p. 22$]$ shows that

$$
\left|\Pi_{1}^{\prime}(z) / \Pi_{1}(z)\right| \leqq c_{2}|z|^{p-1}
$$

for a positive constant $c_{2}$ independent of $z$.

Since

$$
A^{\prime} / A=\left(\Pi_{1}^{\prime} / \Pi_{1}\right)+n a_{n} z^{n-1}
$$

we obtain (3.1) from (3.5) and (3.6).

Now assume that $\theta$ satisfies $0<\left|\theta-\theta_{0}\right| \leqq \varepsilon / 2$. Then by definition of $\delta(P, \theta)$ we have

$$
\left|A\left(r e^{i \theta}\right)\right|=\left|\Pi_{1}\left(r e^{i \theta}\right)\right| \exp \left(\delta(P, \theta) r^{n}\right)
$$

For $r$ sufficiently large the point $\zeta=r e^{i \theta}$ is sufficiently distant from the zeros of $\Pi_{1}$ that (3.4) holds with $R=r$, and so (3.2) and (3.3) hold.

Now, from (3.5) we see that if $r_{0}<r$ are both large then, setting $\psi(s)=\log \left|\Pi_{1}\left(s e^{i \theta}\right)\right|$ we have

$$
\left|\psi(r)-\psi\left(r_{0}\right)\right| \leqq\left|\log \left(\Pi_{1}\left(r e^{i \theta}\right) / \Pi_{1}\left(r_{0} e^{i \theta}\right)\right)\right| \leqq c_{2}\left(r-r_{0}\right) r^{\rho-1}
$$

It then follows that $\left|\psi^{\prime}(r)\right|=O\left(r^{\rho-1}\right)$ as $r \rightarrow+\infty$. Since by (3.7) the function $\phi(r)=\log \left|A\left(r e^{i \theta}\right)\right|$ satisfies the equation

$$
\phi^{\prime}(r)=n \delta(P, \theta) r^{n-1}+\psi^{\prime}(r)
$$

the rest of the lemma follows easily.

Lemma 2. Assume the hypotheses of the Theorem with $\varepsilon$ sufficiently small that $\delta(P, \theta) \neq 0$ for $0<\left|\theta-\theta_{0}\right|<\varepsilon$, and assume that $R(z)$ is analytic and satisfies

$$
|R(z)| \leqq K|z|^{M}
$$


on the sectorial set $A_{0}$ given by

$$
\left|\arg z-\theta_{0}\right| \leqq \varepsilon / 2, \quad|z| \geqq \rho_{0}
$$

where $\rho_{0}$ is large, and $K$ and $M$ are non-negative. For a fixed $a \in A_{0}$ define $H(z)$ in $A_{0}$ by

$$
H(z)=A(z)^{1 / k} \int_{a}^{z} R(t) A(t)^{-1 / k} d t
$$

for some fixed branch of $A(z)^{1 / k}$ in $A_{0}$. Then for some $\rho_{1}>0$ we have the following representation for $H(z)$ in the sectorial set $A_{1}$ given by

$$
\left|\arg z-\theta_{0}\right| \leqq \varepsilon / 4, \quad|z| \geqq \rho_{1}
$$

there exists an analytic function $S(z)$ on $A_{1}$ satisfying

$$
\log ^{+}|S(z)|=O(\log |z|)
$$

in $A_{1}$, and such that for any $\theta$ with $0<\left|\theta-\theta_{0}\right| \leqq \varepsilon / 4$, we have, as $r \rightarrow \infty$,

$$
H\left(r e^{i \theta}\right)=S\left(r e^{i \theta}\right)+c(\theta) A\left(r e^{i \theta}\right)^{1 / k}+O\left(r^{-2}\right)
$$

if $\delta(P, \theta)>0$, where $c(\theta)$ is a constant, while if $\delta(P, \theta)<0$ we have

$$
H\left(r e^{i \theta}\right)=S\left(r e^{i \theta}\right)+O\left(r^{-1}\right)
$$

Proof. In view of (3.1) we may assume that $\rho_{0}$ is so large that

$$
\left|\frac{A^{\prime}(z)}{A(z)}\right| \geqq(c / 2)|z|^{n-1}
$$

on $A_{0}$. We choose $\rho_{1}>\rho_{0}$ so that there is a fixed constant $\varepsilon_{1}$ such that $0<\varepsilon_{1}<1$ and, for each $z$ in $A_{1}$, the closed disc of radius $\varepsilon_{1}|z|$ and centre $z$ is contained in $A_{0}$.

We now define two sequences $\left(R_{m}\right)$ and $\left(S_{m}\right)$ of functions analytic on $A_{0}$ by the equations

$$
R_{1}=R A / A^{\prime}, \quad S_{1}=R_{1}^{\prime},
$$

and for $m \geqq 1$,

$$
R_{m+1}=S_{m} A / A^{\prime}, \quad S_{m+1}=R_{m+1}^{\prime}
$$

Now let $z_{0}=r e^{i \theta}$, with $0<\left|\theta-\theta_{0}\right| \leqq \varepsilon / 4$, belong to $A_{1}$. In view of (3.10) and (3.15), a simple induction using Cauchy's formula for derivatives shows that for each $m=1,2, \ldots$, we have, on $\left|z-z_{0}\right| \leqq \varepsilon_{1}\left|z_{0}\right| 2^{-m}$, the estimate

$$
\left|S_{m}(z)\right| \leqq K_{m}\left|z_{0}\right|^{M-n m}
$$


where $K_{m}$ is a positive constant independent of $z_{0}$. Integration by parts yields, for each $m=1,2, \ldots$,

$$
\begin{aligned}
\int_{a}^{z} R(t) A(t)^{-1 / k} d t & =\left[R_{a}^{z}-k R_{1} A^{-1 / k}+k \int_{a}^{z} S_{1} A^{-1 / k} d t\right. \\
& \vdots \\
& =\left[{ }_{a}^{z} S_{m}^{*} A^{-1 / k}+k^{m} \int_{a}^{z} S_{m} A^{-1 / k} d t\right.
\end{aligned}
$$

where

$$
S_{m}^{*}=-k R_{1}-k^{2} R_{2}-\cdots-k^{m} R_{m}
$$

We now choose $m$ so that $M-n m \leqq-3$. Now if $S_{m} \equiv 0$ we need only set $S=S_{m}^{*}$, while if $S_{m}$ does not vanish identically it remains only to estimate the last integral in (3.19). In the latter case, if $z=r e^{i \theta}$ lies in $A_{1}(3.18)$ and the choice of $m$ imply that

$$
\left|S_{m}(\zeta)\right| \leqq K_{m}|\zeta|^{-3}
$$

for all $\zeta$ in $A_{1}$, and it follows from (3.2) that if $\delta(P, \theta)>0$, the integral

$$
\int_{a}^{\infty} S_{m} A^{-1 / k} d t=c(\theta)
$$

converges, where the path of integration is eventually along the ray $\arg z=\theta$. Thus

$$
\int_{a}^{z} S_{m} A^{-1 / k} d t=c(\theta)-\int_{z}^{\infty} S_{m} A^{-1 / k} d t
$$

and in view of (3.20) and the fact that, by Lemma 1 , the function $\left|A\left(s e^{i \theta}\right)\right|$ is eventually increasing as $s \rightarrow+\infty$, we see that the integral on the right-hand side of (3.21) is bounded by

$$
K_{m}|A(z)|^{-1 / k} \int_{r}^{\infty} s^{-3} d s
$$

The representation (3.13) now holds with $S=S_{m}^{*}$.

Now suppose that $z=r e^{i \theta}$ lies in $A_{1}$, with $\delta(P, \theta)<0$, If $r$ is sufficiently large the point $z^{*}=\sqrt{r} e^{i \theta}$ also lies in $A_{1}$ and we may write

$$
\int_{a}^{2} S_{m} A^{-1 / k} d t=\int_{a}^{z^{*}} S_{m} A^{-1 / k} d t+\int_{z^{*}}^{z} S_{1} A^{-1 / k} d t
$$


where as before we integrate eventually along the ray $\arg z=\theta$. By Lemma $1,\left|A\left(s e^{i \theta}\right)\right|$ is eventually decreasing, so that in view of (3.20) and (3.3) we have, provided that $r$ is large enough,

$$
\left|\int_{a}^{z^{*}} S_{m} A^{-1 / k} d t\right| \leqq B_{1}\left|A\left(\sqrt{r} e^{i \theta}\right)\right|^{-1 / k}
$$

where $B_{1}$ is a positive constant while

$$
\left|\int_{z^{*}}^{z} S_{m} A^{-1 / k} d t\right| \leqq K_{m}\left|A\left(r e^{i \theta}\right)\right|^{-1 / k} \int_{\sqrt{r}}^{r} t^{-3} d t
$$

Since

$$
\log \left|A\left(s e^{i \theta}\right)\right| \sim \delta(P, \theta) s^{n}
$$

as $s \rightarrow+\infty$, using (3.7) and observing that (3.4) will hold with $R=s$ and $\zeta=s e^{i \theta}$, we deduce from (3.23) and (3.24) that (3.14) holds, again with $S=S_{m}^{*}$.

Lemma 3. Suppose that $A(z)$ is analytic in a sector $S$ containing the ray $z=r e^{i \theta}$ and that, for some non-negative $K$ and $n$, and positive $b$ we have

$$
\left|A\left(r e^{i \theta}\right)\right| \leqq K r^{n}
$$

for all $r \geqq b$. Then if $k \geqq 2$, any non-trivial solution $w(z)$ of

$$
w^{(k)}+A(z) w=0
$$

satisfies

$$
\log ^{+}\left|w\left(r e^{i \theta}\right)\right| \leqq M\left(1+r^{(n+k) / k}\right)
$$

for some $M>0$ and for all $r \geqq b$.

Proof. Take $L>0$ and set

$$
v(r)=\exp \left(\int_{b}^{r}\left(L t^{n}\right)^{1 / k} d t\right)
$$

Then (see e.g. $[7$, p. 73]) we clearly have

$$
v^{(k)}(r) / v(r)=L r^{n}+O\left(r^{n-1}\right) \geqq(L / 2) r^{n}
$$

for all $r \geqq b$, provided $L$ is large enough. Now set $h(r)=w\left(r e^{i \theta}\right)$ so that $h$ solves the equation

$$
h^{(k)}(r)-B(r) h=0
$$


where $B(r)=-e^{i k \theta} A\left(r e^{i \theta}\right)$. Choose a positive constant $c$ such that

$$
\begin{gathered}
|h(b)| \leqq c v(b), \\
\left|h^{\prime}(b)\right| \leqq c v^{\prime}(b), \\
\vdots \\
\left|h^{(k-1)}(b)\right| \leqq c v^{(k-1)}(b) .
\end{gathered}
$$

Then, since

$$
|B(r)| \leqq(L / 2) r^{n}
$$

for $r \geqq b$, provided $L$ is large enough, Herold's comparison theorem [8] applies and we deduce that $|h(r)| \leqq c v(r)$ for all $r \geqq b$ and the lemma is proved.

Lemma 4. Suppose that $a(z)$ is analytic in a sector $S$ containing the ray $r e^{i \theta}$ and suppose that $k \geqq 2$ and

$$
\int^{\infty} r^{k-1}\left|a\left(r e^{i \theta}\right)\right| d r<\infty
$$

Then any solution $w(z)$ of

$$
w^{(k)}+a w=0
$$

satisfies

$$
w\left(r e^{i \theta}\right)=0\left(r^{k-1}\right)
$$

as $r \rightarrow+\infty$.

Proof. We may write

$$
w(z)=c_{1}+c_{2} z+\cdots+c_{k} z^{k-1}-\frac{1}{(k-1) !} \int_{e^{t \theta}}^{z}(z-s)^{k-1} a(s) w(s) d s
$$

This gives, for $z=r e^{i \theta}$, with $r>1$, and with $h(z)=w(z) r^{1-k}$,

$$
|h(z)| \leqq O(1)+\frac{1}{(k-1) !} \int_{1}^{r} t^{k-1}\left|a\left(t e^{i \theta}\right)\right|\left|h\left(t e^{i \theta}\right)\right| d t
$$

and we now apply Gronwall's lemma [5, p. 35]. 


\section{Proof of the theorem}

The outline of the proof is as follows. Assuming that (1.2) has a non-trivial solution $f$ with $\lambda(f)<\infty$, we obtain, using the first-order differential equation satisfied by $e^{p}$, a representation $f=W e^{G}$, where $W$ is analytic and of finite order of growth in a sector. Using the asymptotic relations of Lemmas 1 and 2, and the growth estimates of Lemmas 3 and 4 , we then obtain a contradiction.

Suppose then that $f=\Pi_{1} e^{h}$ is a non-trivial solution of (1.2), where $k, A$ and $Q$ are as in the statement (and $Q \equiv 0$ if $\sigma(A)=1$ ), and suppose that $\Pi_{1}$ has finite order. Now (1.2) gives

$$
\left(h^{\prime}\right)^{k}+P_{k-1}\left(h^{\prime}\right)+A+Q=0,
$$

where $P_{k-1}\left(h^{\prime}\right)$ is a differential polynomial of total degree at most $(k-1)$ in $h^{\prime}, h^{\prime \prime}, \ldots$ and with coefficients which are polynomials in $\Pi_{1}^{\prime} / \Pi_{1}, \ldots, \Pi_{1}^{(k)} / \Pi_{1}$, having constant coefficients. Clunie's lemma (see [2]) shows that $\sigma\left(h^{\prime}\right)$ is finite. Differentiating (4.1) we obtain

$$
k\left(h^{\prime}\right)^{k-1} h^{\prime \prime}+Q_{k-1}\left(h^{\prime}\right)+A^{\prime}+Q^{\prime}=0,
$$

where $Q_{k-1}\left(h^{\prime}\right)$ is again a differential polynomial of degree at most $(k-1)$ whose coefficients are polynomials in $\Pi_{1}^{\prime} / \Pi_{1}, \ldots, \Pi_{1}^{(k+1)} / \Pi_{1}$. Multiplying (4.1) by $A^{\prime} / A$ and subtracting from (4.2) we obtain

$$
k R\left(h^{\prime}\right)^{k-1}-\left(A^{\prime} / A\right) P_{k-1}\left(h^{\prime}\right)+Q_{k-1}\left(h^{\prime}\right)-\left(A^{\prime} / A\right) Q+Q^{\prime}=0
$$

where

$$
R=h^{\prime \prime}-\left(A^{\prime} / k A\right) h^{\prime}
$$

From hypothesis (ii) and (3.1) $R$ is analytic and of finite order of growth on a sectorial set given by $\left|\arg z-\theta_{0}\right| \leqq 2 \varepsilon / 3,|z|$ large. Since $\Pi_{1}, A$ and $h^{\prime}$ are all of finite order, standard estimates yield an $N>0$ such that for $j=1, \ldots, k+1$,

$$
\left|A^{\prime} / A\right|+\left|\Pi_{1}^{(j)} / \Pi_{1}\right|+\left|h^{(j+1)} / h^{\prime}\right|=O\left(|z|^{N}\right)
$$

at least outside an $R$-set, and thus (4.5) holds as $z=r e^{i \theta} \rightarrow \infty$ along the ray $\arg z=\theta$ for all $\theta$ outside a set $E_{1}$ of measure zero (see Section 2). By writing $R=h^{\prime}\left(h^{\prime \prime} / h^{\prime}-\left(A^{\prime} / k A\right)\right.$ ) we see that if $\theta \notin E_{1}$ and $r$ is large enough, the inequality $\left|h^{\prime}\left(r e^{i \theta}\right)\right| \leqq 1$ implies that $\left|R\left(r e^{i \theta}\right)\right| \leqq r^{N+1}$. On the other hand, solving (4.5) for $R$, we see that if $\theta \notin E_{1}$, if $r$ is large, and $\left|h^{\prime}\left(r e^{i \theta}\right)\right|>1$, then $\left|R\left(r e^{i \theta}\right)\right| \leqq r^{U}$ for some constant $U$. It now follows from the Phragmén-Lindelöf principle that for some $V>0$, the inequality

$$
\left|R\left(r e^{i \theta}\right)\right| \leqq r^{V} \quad \text { as } r \rightarrow+\infty
$$

holds uniformly in $\theta$ for $\left|\theta-\theta_{0}\right| \leqq \varepsilon / 2$. 
In view of (4.4) we have, for a suitable point $a$, and a constant $K$,

$$
h^{\prime}(z)=A(z)^{1 / k} \int_{a}^{z} R(t) A(t)^{-1 / k} d t+K A(z)^{1 / k}
$$

Now (4.6) implies that the hypothesis of Lemma 2 is fulfilled, and we obtain an analytic function $S(z)$ on the sectorial region $A_{1}$ given by

$$
\left|\arg z-\theta_{0}\right| \leqq \varepsilon / 4, \quad|z| \geqq \rho_{1},
$$

which satisfies (3.12), (3.13) and (3.14), where

$$
H(z)=A(z)^{1 / k} \int_{a}^{z} R(t) A(t)^{-1 / k} d t
$$

We now define $W(z)$ on $A_{1}$ by the equation

$$
f(z)=W(z) A(z)^{\alpha} \exp \left(h(z)-\int_{a}^{z} S(t) d t\right),
$$

where $\alpha=(1-k) / 2 k$. It follows easily from (3.12) and the representation $f=\Pi_{1} e^{h}$, and hypothesis (ii) of the Theorem, that $W(z)$ is analytic and of finite order of growth in $A_{1}$.

Now, in view of (3.12), (4.6) and (3.1), it is easy to see using Cauchy's integral formula that in a sectorial set $A_{2}$ given by $\left|\arg z-\theta_{0}\right| \leqq \varepsilon / 8,|z| \geqq \rho_{2}$, we have, for each $m=0,1, \ldots, k$, and for some $q>0$,

$$
\left|S^{(m)}(z)\right|+\left|R^{(m)}(z)\right|+\left|\left(A^{\prime}(z) / A(z)\right)^{(m)}\right| \leqq|z|^{q}
$$

Now (4.5) and the remark in Section 2 imply that for $\theta$ outside a set $\boldsymbol{E}_{2}$ of measure zero, and for $j=1, \ldots, k$,

$$
\left|\Pi_{1}^{(j)}\left(r e^{i \theta}\right) / \Pi_{1}\left(r e^{i \theta}\right)\right| \leqq r^{N} \quad \text { for } r \geqq r(\theta) .
$$

It now follows from (4.9), (4.10), (4.11) and the representation $f=\Pi_{1} e^{h}$, that if $\theta \notin E_{2}$ and $\left|\theta-\theta_{0}\right| \leqq \varepsilon / 8$, then for $j=1, \ldots, k$, we have

$$
\left|W^{(\theta)}\left(r e^{i \theta}\right) / W\left(r e^{i \theta}\right)\right| \leqq r^{M} \quad \text { for } r \geqq r(\theta)
$$

where $M$ is a fixed constant.

We choose $\theta_{1}, \theta_{2}$ such that $0<\left|\theta_{j}-\theta_{0}\right|<\varepsilon / 8$ and

$$
\delta\left(P, \theta_{1}\right)<0, \quad \delta\left(P, \theta_{2}\right)>0, \quad \text { and } \quad \theta_{2} \notin E_{2} .
$$

We now assert that

$$
W\left(r e^{i \theta_{1}}\right) \rightarrow 0 \quad \text { as } r \rightarrow \infty
$$


We set $d=d_{Q}$ if $Q \not \equiv 0$, and $d=0$ otherwise, and note that by (3.3) and (4.13), it follows that if $\sigma(A)>1$ then Lemma 3 or Lemma 4 applies and we obtain

$$
\log ^{+}\left|f\left(r e^{i \theta_{1}}\right)\right|=O\left(r^{5}\right) \quad \text { as } r \rightarrow+\infty
$$

where $s=(d+k) / k<\sigma(A)$. On the other hand, if $\sigma(A)=1$ (and hence $Q \equiv 0$ ), Lemma 4 applies and we obtain

$$
\log ^{+}\left|f\left(r e^{i \theta_{1}}\right)\right|=O(\log r) \quad \text { as } r \rightarrow+\infty
$$

However, by (3.14), (4.7) and (4.8) we have as $z \rightarrow \infty$ on $\arg z=\theta_{1}$,

$$
h^{\prime}(z)=S(z)+K A(z)^{1 / k}+O\left(|z|^{-1}\right)
$$

so that using (3.3) we have, for $r$ sufficiently large,

$$
h\left(r e^{i \theta_{1}}\right)-\int_{a}^{r e^{i \theta_{1}}} S(t) d t=O(\log r)
$$

Thus from (4.9) we have, for some $b>0$,

$$
W\left(r e^{i \theta_{1}}\right)=f\left(r e^{i \theta_{1}}\right) A\left(r e^{i \theta_{1}}\right)^{(k-1) / 2 k} O\left(r^{b}\right)
$$

as $r \rightarrow+\infty$. But then, using (3.3), (4.15) or (4.16) and the fact that $s<\sigma(A)$, we obtain (4.14) from (4.19).

We now assert that there is a finite, non-zero constant $J$ such that

$$
W\left(r e^{i \theta_{2}}\right) \rightarrow J \quad \text { as } r \rightarrow \infty
$$

We remark that once this claim is established, (4.20) and (4.14) together provide a contradiction as follows. Since $\theta_{1}$ and $\theta_{2}$ satisfying (4.13) can both be chosen arbitrarily close to $\theta_{0}$, the finite order of $W$ in $A$ implies, using the Phragmén-Lindelöf principle, that $W$ is bounded in the sector between the two rays $r e^{i \theta_{1}}, r e^{i \theta_{2}}$ which in turn implies that $J=0$ (see [9, p. 179]), which is impossible.

To establish (4.20), we write $f=W e^{G}$, where, using (4.9),

$$
G^{\prime}=h^{\prime}-S+\alpha\left(A^{\prime} / A\right)
$$

Substituting in (1.2) we obtain an expression of the form

$$
W^{(k)} / W+\sum_{j=0}^{k-1} F_{j}\left(W^{(j)} / W\right)+A+Q=0
$$

where each $F_{j}$ is a polynomial in $G^{\prime}, \ldots, G^{(k)}$, with constant coefficients, satisfying the 
following conditions:

for $j \geqq 2, F_{j}$ has total degree at most $(k-2)$;

$$
\begin{gathered}
F_{1}=k\left(G^{\prime}\right)^{k-1}+B_{1} ; \\
F_{0}=\left(G^{\prime}\right)^{k}+(k(k-1) / 2)\left(G^{\prime}\right)^{k-2} G^{\prime \prime}+B_{2}
\end{gathered}
$$

and where the total degrees of $B_{1}$ and $B_{2}$ are at most $k-2$. We need estimates for the derivatives of $G^{\prime}$ on the ray $\arg z=\theta_{2}$ which we obtain as follows.

From (4.7), (4.8) and (3.13) it follows that for each $\theta$ sufficiently close to $\theta_{2}$ there exists a constant $c_{1}(\theta)$ such that as $r \rightarrow+\infty$,

$$
G^{\prime}\left(r e^{i \theta}\right)=c_{1}(\theta) A\left(r e^{i \theta}\right)^{1 / k}+\alpha\left(A^{\prime}\left(r e^{i \theta}\right) / A\left(r e^{i \theta}\right)\right)+O\left(r^{-2}\right) .
$$

We take a positive $\delta$ so small that the interval $\left|\theta-\theta_{2}\right|<\delta$ lies in $\left|\theta-\theta_{0}\right|<\varepsilon / 8$ and such that $\delta(P, \theta) \geqq \delta_{0}>0$, say, on this smaller interval. Now $G^{\prime} A^{-1 / k}$ has finite order of growth as $z \rightarrow \infty$ in the sectorial set $A_{3}$ given by

$$
\left|\arg z-\theta_{2}\right|<\delta, \quad|z| \geqq \rho_{3},
$$

and from (3.2), (3.1) and (4.26) we see that for each $\theta$ in $\left|\theta-\theta_{2}\right|<\delta$,

$$
G^{\prime}\left(r e^{i \theta}\right) A\left(r e^{i \theta}\right)^{-1 / k} \rightarrow c_{1}(\theta)
$$

as $r \rightarrow+\infty$, so that by the Phragmén-Lindelöf principle $c_{1}(\theta)=c_{1}$ is independent of $\theta$ in this interval. Now $c_{1} \neq 0$ for otherwise we should have, for each $j=1, \ldots, k$, and for some $b_{1}>0$,

$$
\left|G^{(j)}(z)\right|=O\left(|z|^{b_{1}}\right)
$$

for $z$ lying in $\left|\arg z-\theta_{2}\right| \leqq \delta / 2,|z| \geqq \rho_{4}$, using (4.26), (4.10), the Phragmén-Lindelöf principle and Cauchy's estimate. Substituting in (4.22) and using (4.12) we would obtain

$$
\log ^{+}\left|A\left(r e^{i \theta_{2}}\right)\right|=O(\log r)
$$

as $r \rightarrow+\infty$, contradicting (3.2). We deduce from (4.26) that in the sectorial set $A_{2}$ given by $\left|\arg z-\theta_{2}\right| \leqq \delta / 2,|z| \geqq \rho_{5}$ we have

$$
G^{\prime}(z)=c_{1} A\left(r e^{i \theta}\right)^{1 / k}(1+\phi(z))
$$

where $|\phi(z)| \leqq|z|^{-2}$, and, for some $M_{2}>0$, using (4.10),

$$
\left|G^{(j)}\left(r e^{i \theta_{2}}\right)\right| \leqq|z|^{M_{2}}\left|G^{\prime}\left(r e^{i \theta_{2}}\right)\right|
$$

for each $j=2, \ldots, k$, and for all $r \geqq \rho_{6}$, say. 
We proceed to obtain (4.20) using the estimates (4.27) and (4.28). Now, if $r \geqq \rho_{6}$ and $z=r e^{i \theta_{2}}$, we have, using (4.12), (4.22)-(4.25) and (4.27) and (4.28),

$$
k\left(G^{\prime}\right)^{k-1}\left(W^{\prime} / W\right)+\left(G^{\prime}\right)^{k}+c_{k}\left(G^{\prime}\right)^{k-2} G^{\prime \prime}+A=H_{1}
$$

where $c_{k}=k(k-1) / 2$ and

$$
\left|H_{1}(z)\right| \leqq|z|^{M_{3}}\left(\left|G^{\prime}(z)\right|\right)^{k-2}
$$

for some $M_{3}>0$. Now, from (4.26), we may write

$$
\left(G^{\prime}\right)^{k}=c_{1}^{k} A+k c_{1}^{k-1} A^{(k-1) / k}\left(\alpha\left(A^{\prime} / A\right)+O\left(r^{-2}\right)\right)+H_{2}
$$

and

$$
\left(G^{\prime}\right)^{k-2}=c_{1}^{k-2} A^{(k-2) / k}+H_{3}
$$

where for some $M_{4}>0$, and for all $z=r e^{i \theta_{2}}$ with $r \geqq \rho_{7}$, say

$$
\left|H_{2}(z)\right| \leqq|z|^{M_{4}}\left(\left|G^{\prime}(z)\right|\right)^{k-2}
$$

and

$$
\left|H_{3}(z)\right| \leqq|z|^{M_{4}}\left(\left|G^{\prime}(z)\right|\right)^{k-3}
$$

unless $k=2$, in which case $H_{3} \equiv 0$.

We need a more precise estimate for $G^{\prime \prime}$ than (4.28). Now (4.26) and the PhragménLindelöf principle imply that $G^{\prime}-c_{1} A^{1 / k}$ is analytic and bounded by a power of $|z|$ in a sector about the ray $r e^{i \theta_{2}}$, so that Cauchy's formula for derivatives yields

$$
G^{\prime \prime}=\left(c_{1} / k\right) A^{(1-k) / k} A^{\prime}+H_{4}
$$

for $z=r e^{i \theta_{2}}$ and $r \geqq \rho_{8}$, say, where

$$
\left|H_{4}(z)\right| \leqq r^{M_{5}}
$$

for some $M_{5}>0$. Substituting (4.31), (4.32) and (4.35) in (4.29) we obtain

$$
\begin{aligned}
& k\left(G^{\prime}\right)^{k-1}\left(W^{\prime} / W\right)+\left(c_{1}^{k}+1\right) A+k c_{1}^{k-1} A^{(k-1) / k}\left(\alpha\left(A^{\prime} / A\right)+O\left(r^{-2}\right)\right) \\
& \quad+c_{k}\left(c_{1}^{k-2} A^{(k-2) / k}+H_{3}\right)\left(\frac{c_{1}}{k} A^{(1-k) / k} A^{\prime}+H_{4}\right) \\
& =H_{1}-H_{2} .
\end{aligned}
$$


Using (4.27), (4.30), (4.33), (4.34) and (4.36) we obtain, noting that $c_{k}=k(k-1) / 2$,

$$
\begin{aligned}
& k\left(G^{\prime}\right)^{k-1}\left(W^{\prime} / W\right)+\left(c_{1}^{k}+1\right) A \\
& +k c_{1}^{k-1} A^{(k-1) / k}\left(\alpha\left(A^{\prime} / A\right)+O\left(r^{-2}\right)\right) \\
& +\frac{k-1}{2} c_{1}^{k-1} A^{(k-1) / k}\left(A^{\prime} / A\right)=H_{5}
\end{aligned}
$$

where

$$
\left|H_{5}\left(r e^{i \theta_{2}}\right)\right| \leqq r^{M_{6}}\left(\left|G^{\prime}\left(r e^{i \theta_{2}}\right)\right|\right)^{k-2}
$$

for some $M_{6}>0$ and all $r \geqq \rho_{9}$, say.

But $\alpha=(1-k) / 2 k$ and we therefore have

$$
k\left(G^{\prime}\right)^{k-1}\left(W^{\prime} / W\right)+\left(c_{1}^{k}+1\right) A+A^{(k-1) / k} O\left(r^{-2}\right)=H_{5} .
$$

Now, (3.2), (4.27), (4.37) and (4.12) imply that $c_{1}^{k}+1=0$. The same estimates now imply that $\left(W^{\prime} / W\right)=O\left(r^{-2}\right)$ in (4.38) and (4.20) is proved.

Acknowledgement. This work was supported in part by NSF grant DMS 8420561 .

\section{REFERENCES}

1. S. Bank, G. Frank and I. Laine, Uber die Nullstellen von Lösungen linearer Differential gleichungen, Math. Z. 183 (1983), 355-364.

2. S. BANK and I. LAINE, On the growth of meromorphic solutions of linear and algebraic differential equations, Math. Scand. 40 (1977), 119-126.

3. S. BANK and I. LAINE, On the oscillation theory of $f^{\prime \prime}+A f=0$ where $A$ is entire, Trans. Amer. Math. Soc. 273 (1982), 351-363.

4. S. Bank, I. Laine and J. K. Langley, On the frequency of zeros of solutions of second order linear differential equations, Resultate Math., to appear.

5. R. Bellman, Stability Theory of Differential Equations (McGraw-Hill, New York, 1953).

6. W. K. Haymen, Slowly growing integral and subharmonic functions, Comment. Math. Helv. 34 (1960), 75-84.

7. W. K. Hayman, Meromorphic Functions (Oxford at the Clarendon Press, 1964).

8. H. Herold, Ein Vergleichssatz für komplexe lineare Differential gleichungen, Math. Z. 126 (1972), 91-94.

9. E. C. Titchmarsh, The Theory of Functions (Oxford, 1949).

Department of Mathematics

UNIVERSITY OF ILLINOIS

1409 West Green St.

URBANA 61801, USA
Department of Pure Mathematics UNIVERSITY OF ST. ANDREWS NorTh HAUGH St. Andrews KY16 9SS SCOTLAND 